\title{
THE ORIGIN OF THE SLOWLY VARYING COMPONENT
}

\author{
W. N. CHRISTIANSEN AND D. S. MATHEWSON \\ Radiophysics Laboratory, Commonwealth Scientific and Industrial \\ Research Organization, Sydney, Australia
}

Two main mechanisms have been proposed to explain the origin of the slowly varying component [1] of the decimeter-wavelength radiation from the sun. Both theories $[2,3,4]$ are based on the facts that $(a)$ this component of the sun's radiation is relatively steady in output for periods of days; $(b)$ it originates from active regions on the sun; and $(c)$ its magnitude is closely related to the sunspot area in the region.

The first theory put forward [2,3] was that the magnetic fields of the sunspots raise the emitting region's height (extraordinary mode) from the cooler chromosphere into the hot corona. The second theory $[4,5]$ is that the sources are hot, dense regions in the corona. An explanation that combines the effects of hot, dense regions and magnetic fields has also been suggested [6].

The first mechanism would be expected to produce some degree of circular polarization in the radiation; the second would not.

The difficulty of testing the various mechanisms has been the lack of essential information on brightness temperature, size, and position of the regions of origin in the solar atmosphere. Optical observations of the corona are possible only at the limb of the sun. In the past, radio observations, apart from rare eclipse measurements, have been inadequate in resolution; in recent years, however, high-resolution measurements have been undertaken.

High-resolution radio observations that use the new crossed-multiple interferometer [7], which operates at a $20 \mathrm{~cm}$ wavelength and scans the sun with a 3 minute-of-arc pencil beam, were begun in Sydney in 1957 June. The observations are a step further in the study of the slowly varying radioemission sources that was begun [8] with a strip-scanning multiple interferometer during the minimum phase of the present sunspot cycle.

The new observations show the regions of origin of the slowly varying component at a $20-\mathrm{cm}$ wavelength to be at, or near, normal coronal temperatures. They are the same size and shape as the chromospheric plages, which they invariably overlie at heights of some tens of thousands of kilometers. Within the accuracy of measurements, the radiation shows no circular polarization. Consequently, this indicates the radiation's thermal mode of origin in regions considerably denser than the surrounding corona but with similar temperatures.

When observations at other wavelengths are taken into account, it appears that these dense regions extend in columns-perhaps constrained from expan- 
(o)

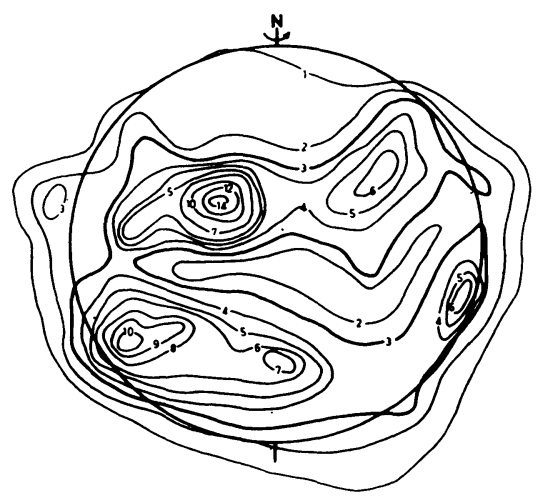

(c)

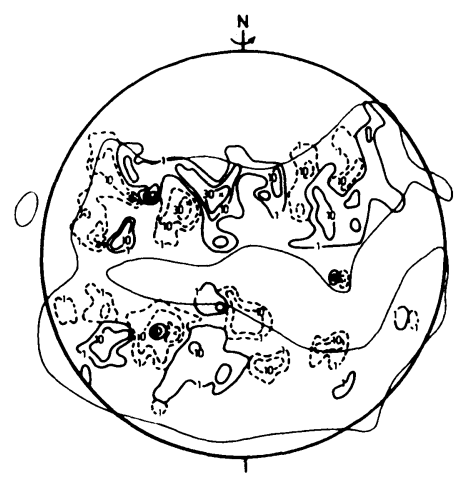

(b)

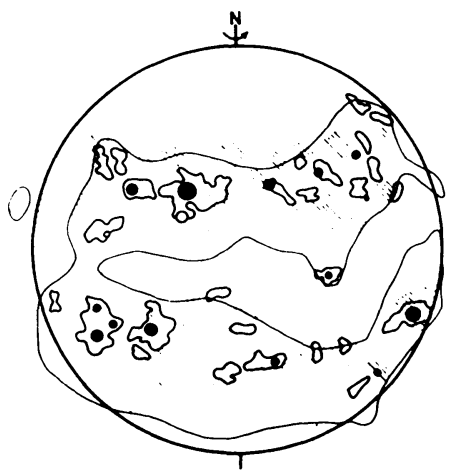

Fig. 1. Solar observations on 1957 November 11 (corrected for solar rotation to a common time at $03^{\mathrm{h}} 00^{\mathrm{m}}$ U.T.). (a) Radio brightness contours $(\lambda=20 \mathrm{~cm})$. The contour unit is $7.5 \times 10^{4}{ }^{\circ} \mathrm{K}$. (b) Fraunhofer Institut solar map showing plage and position of sunspot groups. (c) Mt. Wilson magnetogram of sun, redrawn in contour form. Contours are at 1 , 10,30 gauss; dotted lines, south polarity ; full lines, north polarity. $[\operatorname{In}(b)$ and $(c)$, no. 3 of the radio brightness contours $\left(2.2 \times 10^{5}\right.$ ${ }^{\circ} \mathrm{K}$ ) has been superimposed on the maps to facilitate comparisons of position and shape].

sion by local magnetic fields-from the chromosphere out to at least $100,000 \mathrm{~km}$ in the corona and may form the base of the great coronal streamers seen during solar eclipses.

It seems probable that all the variable solar phenomena seen by radio observers occur in these regions of high electron density.

\section{POSITION, SIZE, AND SHAPE OF THE RADIO SOURCES}

A study of the daily radio maps of the sun has shown that the radioemitting sources lie above the active regions on the sun and that their shape is delineated in a lower part of the solar atmosphere by plage faculaire, which invariably accompanies each radio source.

In Fig. 1 the three maps show contours of radio brightness, chromospheric plage (Fraunhofer Institut), and magnetic field intensity (Babcock) determined from solar observations on the same day. The correspondence in size and shape of the sources of radio emission, the areas of localized magnetic field, and the plage is clearly seen. It appears that the peak of radio emission 
lies above the brightest plage area and not above the peak of magnetic-field strength, which coincides with the sunspots. This correspondence has been found on all days for which we have made a comparison.

At the present part of the solar cycle, the radio sources typically subtend an angle of about 5 minutes of arc at the earth, i.e. they extend over about $250,000 \mathrm{~km}$.

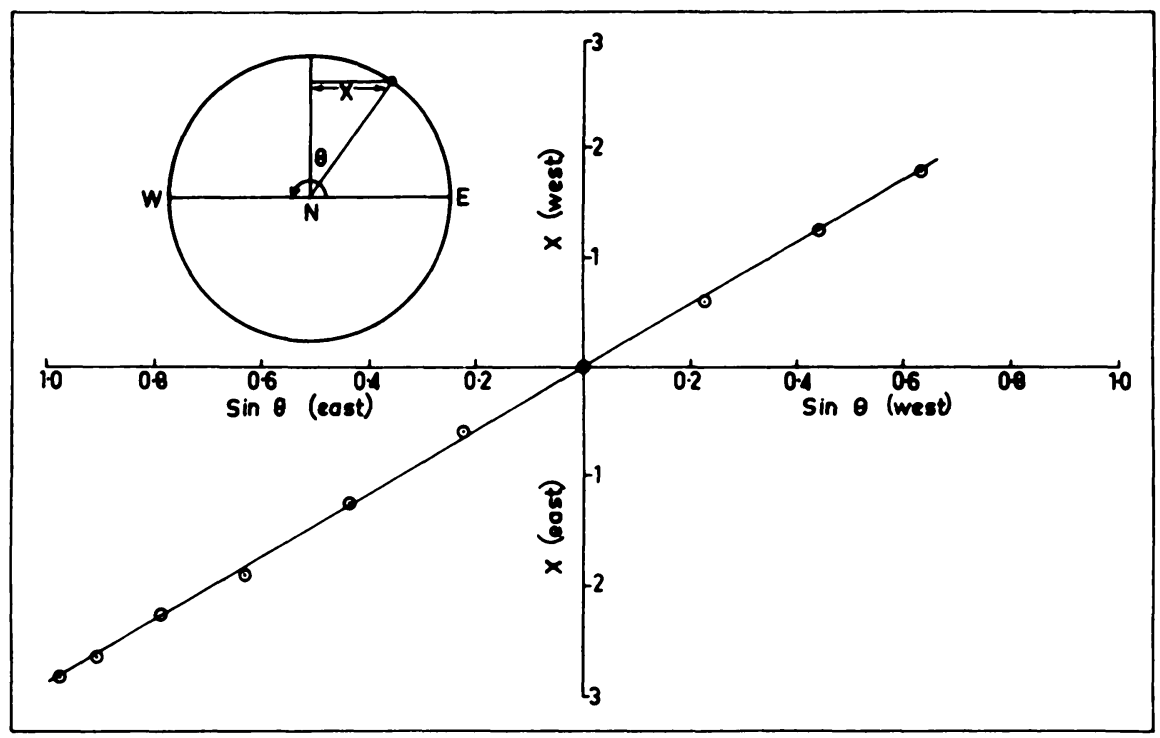

FIG. 2. The passage of a radio source (C.M.P. 1957 October 26, lat. 23 degrees S) across the disk of the sun. The displacement of the source from the central meridian on the disk is plotted against the sine of the angle of rotation of the sun.

The sources move across the disk more rapidly than the underlying photosphere because of their greater height. It is possible, with certain precautions, to use this rate of travel to determine the height of the sources. We have done this for a number of regions and have found that their heights varied between 20,000 and $100,000 \mathrm{~km}$ with estimated errors of $\pm 10,000 \mathrm{~km}$. The average height was about $40,000 \mathrm{~km}$. A typical height-determination is illustrated in Fig. 2.

In a previous paper [8] dealing with solar observations at a time of sunspot minimum, it was shown that the radio flux from a source followed a cosine law as it passed from the central meridian to the limb.

This suggested that the emitting surface's form was that of a disk lying parallel to the photospheric surface. Our recent observations show a similar variation and confirm the previous conclusion. Confirmation of the disk-like shape of an emitting region is found also in the change in shape-a reduction in apparent width-as the region approaches the limb of the sun.

To sum up, the emitting regions are situated at heights of 20,000 to $100,000 \mathrm{~km}$ above the chromospheric plage; their extension in latitude and 
longitude is similar to that of plage and also to that of localized magnetic fields observed by Babcock in the lower parts of the sun's atmosphere; their extension in height is small compared with their extension parallel to the photosphere.

\section{DEVELOPMENT OF ACTIVE REGIONS}

Radio pictures of the sun at intervals of its rotation period are shown in Fig. 3 over a period of four months. They show that the slowly varying regions have lifetimes of the order of three months and exhibit their own movements, expanding, contracting, and changing in size but remaining essentially in the same region on the sun. No systematic trend in height, intensity, or size with age of the region has, so far, been found.

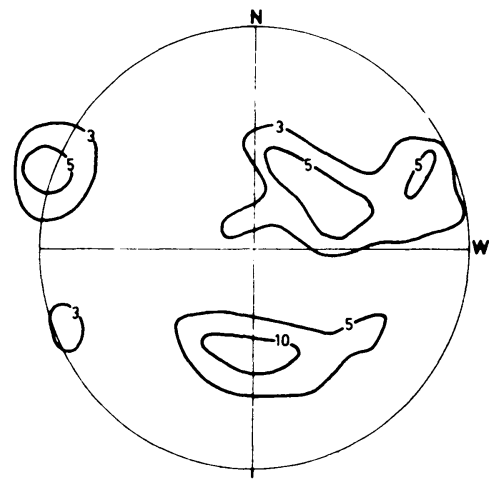

1957 OCT 18

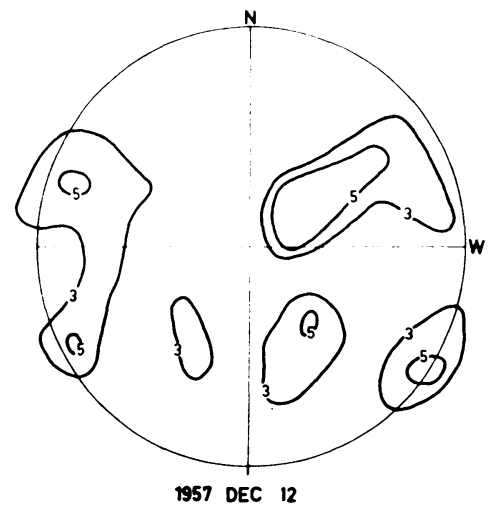

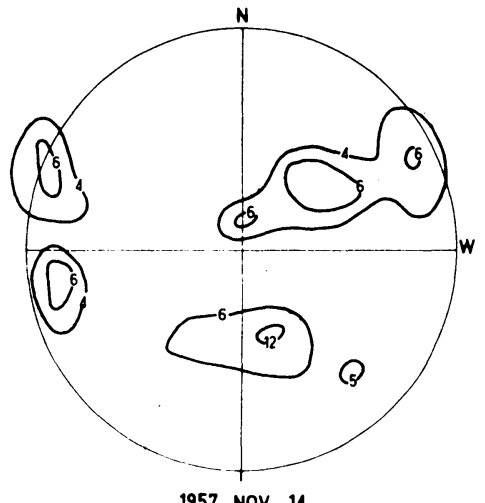

1957 NOV 14

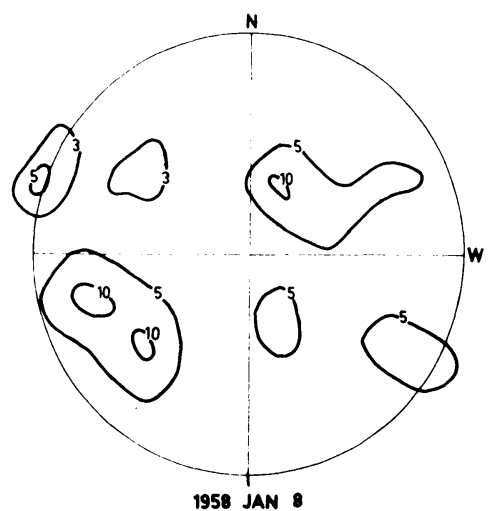

Fig. 3. Radio maps of the sun at intervals of one solar rotation. The brightness contours are marked in units of $10^{5} \mathrm{~K}$.

\section{BRIGHTNESS TEMPERATURE}

The most significant new information relates to brightness temperature. In Fig. 4, the brightness temperature of some 60 sources is plotted. The temperatures range from small values up to a cut-off at about $1.5 \times 10^{8}{ }^{\circ} \mathrm{K}$, the 


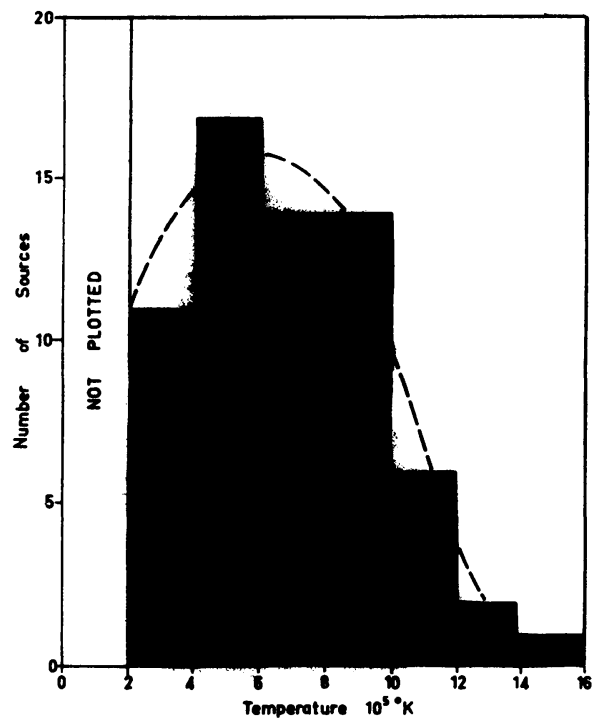

FIg. 4. The distribution of the peak brightnesses of 65 radio sources observed from 1957 September to 1958 January. The beamwidth of the aerial is 3 minutes of arc. most commonly found value being at $0.6 \times 10^{\circ}{ }^{\circ} \mathrm{K}$. (The temperatures plotted do not include these associated with short-lived disturbances on the sun).

The sharp upper limit to the brightness-temperature range is at a value near the commonly accepted coronal temperature; this is strong evidence that the radio sources are simply optically dense parts of the corona. Such regions could be condensations of high electron density, or alternatively, the high optical density could be the effect of magnetic fields.

It may be noted that the previous works $[8,9]$ in which higher temperatures have been suggested were based on what is almost certainly an underestimate of the size of the emitting region. This was the result of the limitations of the measurement technique. The most commonly found temperature in the present work is $0.6 \times 10^{\circ} \mathrm{K}$, which is lower than the accepted coronal temperature. This may well be due to an overestimate in the size of the emitting region owing to a nonuniform density distribution in the region.

\section{POLARIZATION}

If the magnetic field alone were responsible [2,3] for the optical thickening of the highly emitting regions, it might be expected to give rise to the emission of circularly polarized radiation. It is known that this is observed [10] at a wavelength of $3 \mathrm{~cm}$ and that small effects are found at $10 \mathrm{~cm} \mathrm{[11,}$ 12]. To investigate the $20-\mathrm{cm}$ radiation, we rotated the dipoles of the northsouth line of aerials, so that they were mutually perpendicular to those of the east-west line of aerials. An alternating phase change of \pm 90 degrees was introduced into the output from one line of aerials before we combined it with the output from the other line. The alternating component of the combined output represented the difference of the left-and the right-hand circularly polarized components of the radiation in the aerial beam, and was a sensitive detector of such radiation. It was found that during a one-month period no circularly polarized component greater than 2 per cent of the randomly polarized emission was seen. This agrees with earlier findings (unpublished) at sunspot minimum and indicates that the simple magneto-ionic explanation [2, 3], i.e. that the sunspot field raises the level of origin from the chromosphere into the high-temperature corona, cannot be sustained.

However, a gyro theory involving harmonics of the gyroresonance fre- 
quency was recently [13] put forward. In this mechanism it is claimed that circularly polarized radiation may not be detected in the received radiation. Hence, our experimental results cannot eliminate completely the possibility of gyro effects, although they give no support to this explanation.

Attempting to find possible magnetic effects, we looked for correlations between the magnetic-field intensity of the spot group (Mt. Wilson data) and the brightness or height of the radio emission in the region above. For the limited number of sources investigated, no correlation was found.

\section{EVIDENCE FOR HIGH DENSITIES}

There is considerable evidence from optical observations that high coronal densities are found above the optically active regions on the sun. (The optical observations are limited, of course, to regions off the solar limb.) At solar eclipses and by using Lyot's coronagraph, observers have been able to measure the brightness of the corona, both in white light and in the light of the coronal emission lines. The $\mathrm{K}$-corona seen in white light gives a direct measure of electron density, whereas the brightness of the emission lines, which depends on both temperature and density, is more difficult to interpret in terms of density.

Of the many investigations, two long-term ones may be mentioned. Trellis [14] has demonstrated that a coronal jet, as seen in the green coronal line, normally is found above chromospheric plage. He interprets this as an increase in temperature and density above the plage. Waldmeier [5] has made a long and thorough study of what he called "coronal condensations," which can be seen both in white light and in the light of the coronal emission lines. The condensations appear above sunspot groups.

In the condensations, the electron density is about 20 times greater than at similar levels in the undisturbed corona. Using Waldmeier and Muller's model [4] for a condensation, Pawsey and Smerd [15] have shown that the spectral-intensity distribution of thermal emission from such a condensation is in reasonably good agreement with observation. This model gives a height of origin for the $20-\mathrm{cm}$ radiation which fits our new results, but the source size is too small and it seems that the hemispherical model will require modification.

Since there is good evidence of high coronal densities above active regions, there appears to be little point in explaining the $20-\mathrm{cm}$ radiation in terms other than of thermal emission from regions of high electron density in the corona.

At the shorter wavelengths, especially at $3 \mathrm{~cm}$, there is evidence of some degree of circular polarization in the emitted radiation. This indicates that at the short wavelengths, for which the electron density necessary to make the region opaque is higher than at $20-\mathrm{cm}$ wavelength, and for which, therefore, the region of origin should be in the lower and cooler parts of the solar atmosphere, the local magnetic fields may raise the level of the extraordinary component of the radiation into the higher and hotter regions; this will cause a predominance of one circularly polarized component. 
More information on the $3-\mathrm{cm}$ emissions is required, however, before any detailed model of the lower part of the dense regions can be derived. For example, the source sizes for both the circularly polarized and the circularly unpolarized components must be determined.

The close association found by Babcock [16] between chromospheric plage and localized magnetic fields in the photosphere, and the association of these with the dense regions of the radio sources, suggests that the field plays some important part in producing these regions of high electron density. Although the fields are measured only in the photosphere, one may presume, on the basis of observed movements of prominence material, that the fields penetrate into the corona. It would seem probable, therefore, that the field provides a magnetic " bag" that prevents the hot, dense material from dispersing.

The shape of these dense regions appears to be that of a vertical column, with its base in the photosphere, and presumably having a rapid change of electron density with height. This shape is suggested by the close correspondence in size and shape between the sources of $(a)$ surface magnetic fields, (b) chromospheric plage, and (c) radio sources (at $\lambda=20 \mathrm{~cm}$ ) with heights up to $100,000 \mathrm{~km}$. Additional information, which is available for radio emission in the $\mathrm{cm}$ range of wavelengths $[17,20,21,22]$, is completely consistent with the correspondence in shape and size of radio sources and plage.

The explanation of the slowly varying component of the sun's radio emission in terms of thermal emission from high-density regions, at normal coronal temperatures, fits well with the very high correlation found by Denisse and Kundu between the ionization in the E-region of the earth's atmosphere and the slowly varying component of the solar decimeter wavelength. The connection between plage and the ionizing radiation was demonstrated earlier by Allen [19]. At least part of the sun's X-ray emission, which produces the E-layer, would vary with the coronal density in the same way (i.e., in proportion to the square of the electron density) as the radio emission. C. W. Allen, in fact, has used the term "slowly varying component" to describe the part of the sun's ionizing radiation that produces terrestrial effects that show time variations similar to those of the decimeter wavelength radiation.

\section{CONCLUSION}

It has been shown that the slowly varying component of solar radio emission appears to originate thermally from high-density regions in the corona situated at heights from 20,000 to $100,000 \mathrm{~km}$ above areas in which appear plages and magnetic fields. The regions are at normal coronal temperatures. The close correlation between the plage areas and size of the $20-\mathrm{cm}$ radio sources is remarkable, and the information available at $\mathrm{cm}$ wavelengths shows that this correlation is true also for the shorter wavelengths. It appears then that the cross-section of the dense regions is fairly constant with height and is delineated by the plage in the lower regions of the sun's atmosphere. It is interesting to speculate whether the mechanism that pro- 
duces the plage also produces the dense region, or whether the dense region, by virtue of intense back radiation in the ultra-violet and $\mathrm{X}$-regions of the spectrum, itself produces the plage.

The emitting regions extend over wide areas on the solar disk, but the extension in height of a source observed at $\lambda=20 \mathrm{~cm}$ is small compared with its width. The latter observation may be simply the result of a rapid change of electron density with height.

It is possible that these regions extend right out to form the coronal streamers. High-resolution radio observations at meter wavelengths [23] at the limb of the sun will help solve this point. Significant information will also be given by the combination of directional and spectral observations [24] at meter wavelengths now being conducted in Sydney.

To obtain a complete model of these regions it is necessary for the same region to be studied with highly directional arrays operating over a wide range of wavelengths. In the world today there exists a number of arrays that have high resolving power in at least one direction. These operate at wavelengths of $3,7,10,20,88$, and $176 \mathrm{~cm}$, which form a series ideal for this purpose. Since the component of the sun's radiation to be studied is slowly varying, truly simultaneous observations are not required. Observers from six nations have recently combined their radio and optical results on spot group 12808 (Mt. Wilson number). In this way, the extent of a region, its density, temperature, and magnetic-field gradients may be determined. It is hoped that the combination of these radio results with $\mathrm{H} \alpha, \mathrm{K} 3, \mathrm{~K}$-corona, and Mt. Wilson magnetic observations will result in a further step toward understanding this important solar feature.

\section{REFERENCES}

[1] Pawsey, J. L., and Yabsley, D. E. Aust. J. Sci. Res. A 2, 198, 1949.

[2] Lehany, F. J., and Yabsley, D. E. Aust. J. Sci. Res. A 2, 48, 1949.

[3] Denisse, J. F. Ann. Astrophys. 13, 181, 1950.

[4] Waldmeier, M., and Müller, H. Z. Ap. 27, 58, 1950.

[5] Waldmeier, M. Z. Ap. 40, 221, 1956.

[6] Piddington, J. H., and Minnett, H. C. Aust. J. Sci. Res. A 4, 131, 1951.

[7] Christiansen, W. N., Mathewson, D. S., and Pawsey, J. L. Nature, 180, 944, 1957.

[8] Christiansen, W. N., Warburton, J. A., and Davies, R. D. Aust. J. Phys. 10, 491, 1957.

[9] Christiansen, W. N., Yabsley, D. E., and Mills, B. Y. Aust. J. Sci. Res. A 2, 506, 1949.

[10] Kaidanovskii, N. L., Korol'kov, D. V., Soboleva, N. S., and Khaikin, S. E. Dokl. Akad. Nauk S.S.S.R. 112, 1012, 1957.

[11] Covington, A. E. Proc. I.R.E. 37, 407, 1949.

[12] Kakinuma, T. Proc. Res. Inst. Atm. Nagoya U. 4, 78, 1956.

[13] Twiss, R. Q. Unpublished data.

[14] Trellis, M. Ann. Astrophys. Supplement No. 5, 1957.

[15] Pawsey, J. L., and Smerd, S. F. The Solar System (ed. G. Kuiper). Vol. I, Chapter 7, 1953.

[16] Babcock, H. W., and Babcock, H. D. Ap. J. 121, 349, 1953. 
[17] Alon, I., Kundu, M. R., and Steinberg, J. L. C.R. 244, 1726, 1957.

[18] Denisse, J. F., and Kundu, M. R. C.R. 244, 45, 1957.

[19] Allen, C. W. Terr. Magn. Atmos. Elect. 53, 433, 1948.

[20] Dodson, Helen W. Ap. J. 119, 564, 1954.

[21] Hatanaka, T., Akabane, K., Moriyama, F., Tanaka, H., and Kakinuma, T. Publ. Astr. Soc. Japan, 7, 161, 1955.

[22] Vitkevich, V. V., Kuz'min, A. D., and Salomonovich, A. E. Dokl. Akad. Nauk S.S.S.R. 118, 1091, 1958.

[23] Blum, E. J., Boischot, A., and Ginat, M. C.R. 243, 19, 1956.

[24] Wild, J. P., and Sheridan, K. V. Proc. I.R.E. 46, 160, 1958.

\section{Discussion}

Vitkevich and Mathewson (submitted 1958 September 12): During this symposium we have compared some $20-\mathrm{cm}$ and $3-\mathrm{cm}$ sular radio maps for a period 1957 July 18 to 26 and have found a good correspondence in the position, size, and shape of the emission regions at these two wavelengths and the underlying plages which are invariably associated with these sources. Fig. 1 shows the brightness contours at $3-\mathrm{cm}$ and $20-\mathrm{cm}$ wavelength, together with an $\mathrm{H} \alpha$ picture (Sydney) for 1957 July 22.

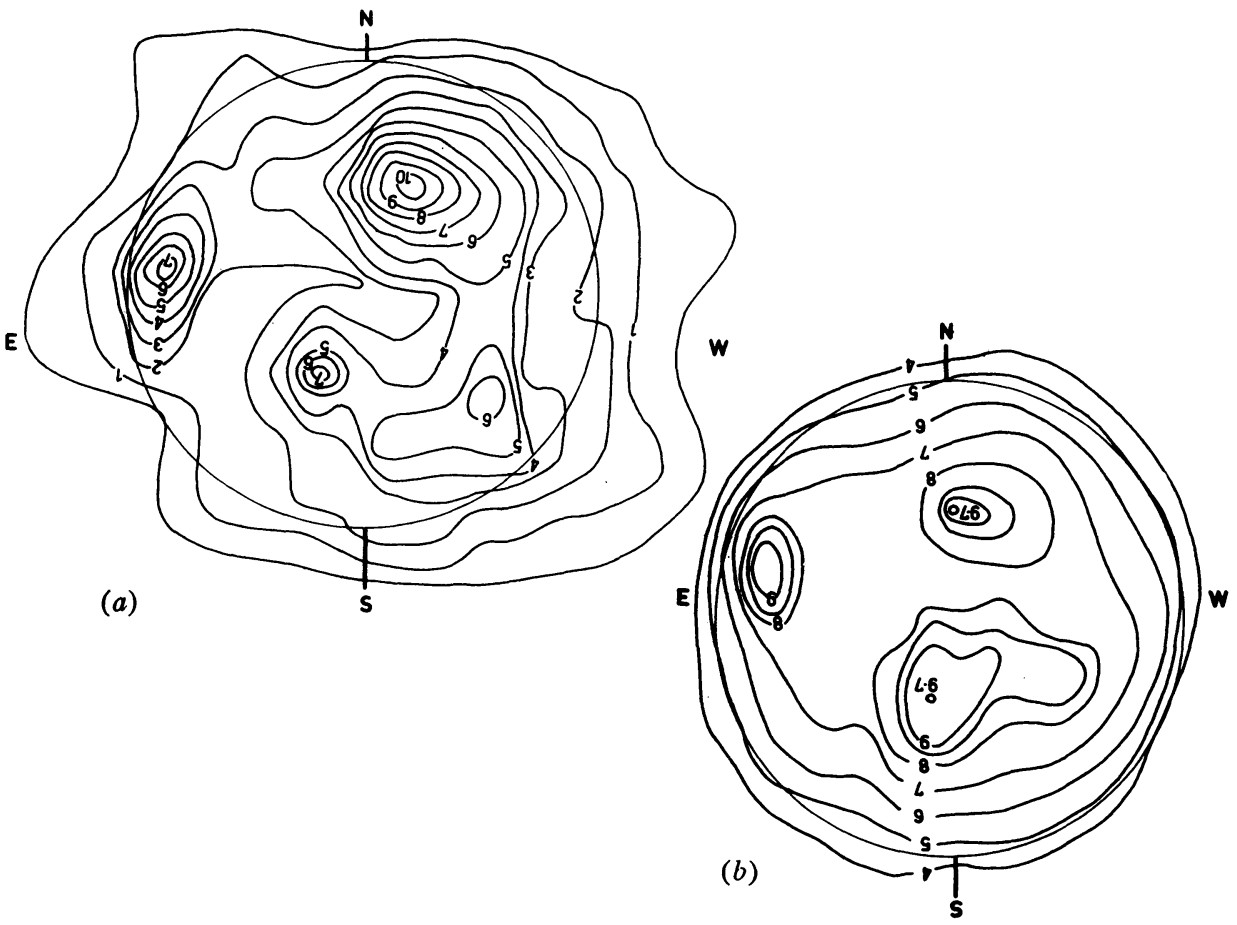

FIG. 1. (a) Brightness contours at $21-\mathrm{cm}$ wavelength, 1957 July $22^{\circ} 02^{\mathrm{h}} 00^{\mathrm{m}}$ U.T. Color brightness unit $=8.5 \times 10^{4}{ }^{\circ} \mathrm{K}$. (b) Brightness contours at 3-cm wavelength, 1957 July 22. (c) $\mathrm{H} \alpha$ picture (Sydney), 1957 July 22. 


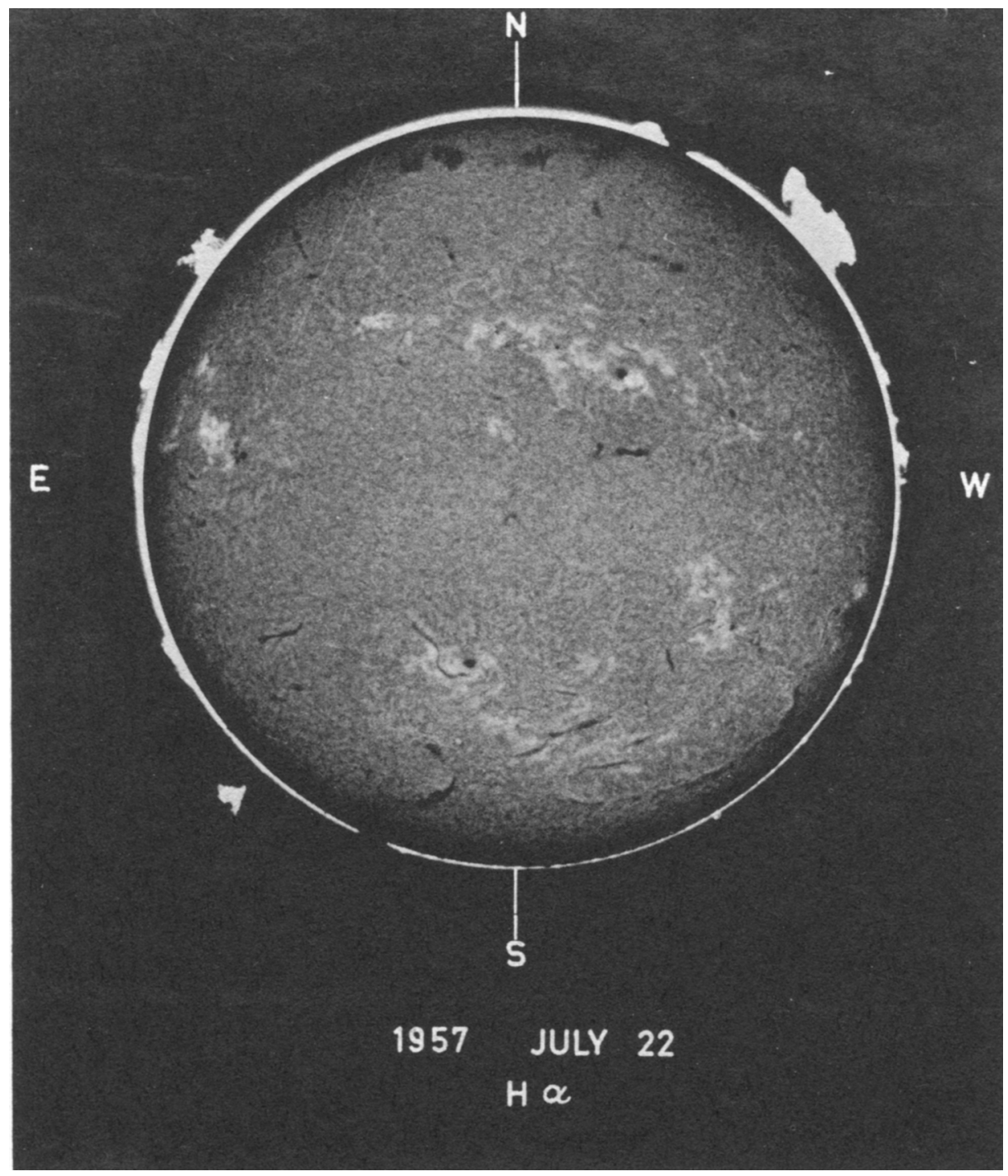

FIG. 1. (c) 